\title{
Drag resolution of a PIV wake rake for transiting models
}

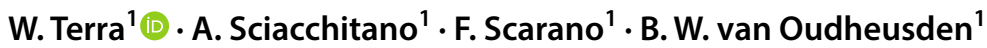

Received: 15 March 2018 / Revised: 31 May 2018 / Accepted: 8 June 2018 / Published online: 29 June 2018

(c) The Author(s) 2018

\begin{abstract}
The accuracy of the "PIV wake rake" method to measure the drag of transiting objects is evaluated. Tomographic particle image velocimetry measurements are conducted on a sphere towed at different speeds, within a Reynolds number range where the drag coefficient is constant. In contrast to PIV wake rake application on steady models in wind tunnels, where the upstream conditions can be accurately controlled and known a priori, measurement of the flow field prior to the passage of the model is essential for an accurate estimation of the drag for towed models when control of the undisturbed conditions is more challenging. The drag resolution of the technique is estimated to prospect the use of the technique in large-scale applications. A resolution of approximately 20 drag counts is obtained which is coarser than wind tunnel experiments but comparable to techniques used for field measurements.
\end{abstract}

\section{Graphical abstract}

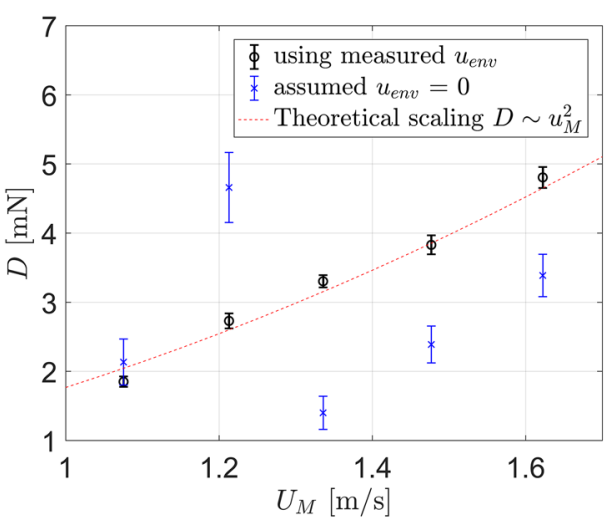

The wake rake has a long tradition as a practical instrument for measuring the aerodynamic drag of objects immersed in a stream. Originally, the resistance force was derived from the pressure field measured in the wake by a Pitot-tube rake, by invoking the conservation of momentum in a control volume containing the object (e.g., Jones 1936). The approach is often used to estimate the aerodynamic drag of airfoils (e.g., Selig et al. 2011) or three-dimensional models (e.g., Brune 1994; Maskell 1973) in a stationary configuration.

W. Terra

w.terra@tudelft.nl

1 Aerospace Engineering Department TU Delft, Delft, The Netherlands

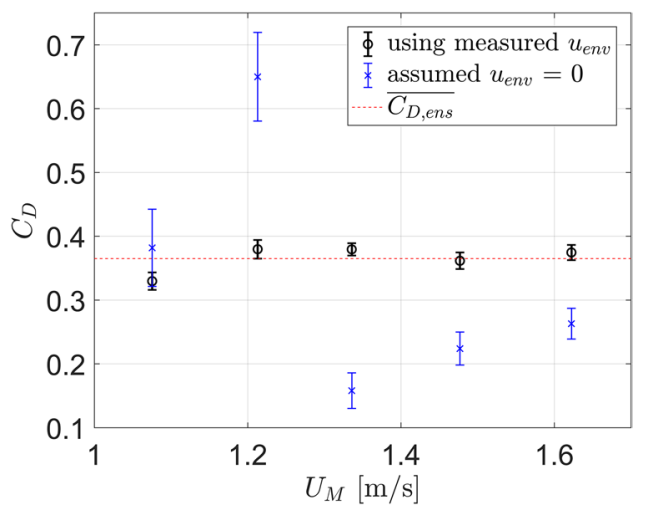

This approach offers the advantage of more sensitivity compared to force balances when the drag force is small (e.g., Selig et al. 2011). In addition to the drag force, the wake rake yields the velocity distribution behind the model, which relates the aerodynamic loads with the flow structure in the wake of the model.

More recently, particle image velocimetry (PIV) measurements have been used as an alternative to the Pitot-tube wake rake. The "PIV wake rake" returns the mean and instantaneous aerodynamic loads on stationary models in wind tunnels, as demonstrated by Van Oudheusden et al. (2007) and De Kat and Bleischwitz (2016), among others. Furthermore, the use of PIV as wake rake on transiting models has been introduced in the past to study the structure of aircraft 
trailing vortices in towing tank experiments (Scarano et al. 2002) as well as behind a catapulted model (Von Carmer at al. 2008). More recently, the quantitative measurement of mean aerodynamic loads on a towed sphere in air has been demonstrated with the use of tomographic PIV (Terra et al. 2017). In the latter work, helium-filled soap bubbles (HFSB, Bosbach et al. 2009) were used as flow tracers to obtain a measurement volume significantly larger than that achievable with micron-sized droplets, preluding an upscale of the technique for application in, e.g., the automotive industry and speed sport. In the latter case, the method may be used to detect the effect of small modifications of the model geometry or surface roughness on the aerodynamic drag (e.g., Brownlie et al. 2016), and, hence, an understanding of its drag resolution is of crucial importance. This information is not reported by Terra et al. (2017) and neither is it in other literature. Moreover, a thorough discussion of the parameters governing the uncertainty of the aerodynamic drag obtained from the PIV wake rake for a transiting object, in particular the role of the non-quiescent flow conditions prior to the model transit, is missing.

The present work aims to quantify the drag resolution of the PIV wake rake by evaluating the aerodynamic drag of a sphere at different values of the towing velocity $U_{M}=\{1.08$; $1.21 ; 1.34 ; 1.48 ; 1.62\} \mathrm{m} / \mathrm{s}$. The drag coefficient of a sphere exhibits a practically constant value over the flow regime $1000<\operatorname{Re}<200,000$ (Schlichting 1979). Hence, in the present range of conditions $(6600<R e<11,400)$ the aerodynamic drag is expected to follow a quadratic increase with model speed. This is exploited to estimate the accuracy of the drag from the PIV wake rake, first for a single-passage measurement considering a finite time interval of the velocity measurement. Furthermore, the drag measurement obtained from averaging the results from repeated passages is evaluated. The influence of non-quiescent flow conditions prior to the passage is taken into account and discussed and, finally, the drag resolution of the PIV wake rake for transiting models is compared to that of other techniques to appreciate its applicability.

The experimental apparatus and procedures follow those of previous investigations (Terra et al. 2017). Tomographic PIV measurements are performed upstream and downstream of a sphere of diameter $d=10 \mathrm{~cm}$ which is towed through a rectangular channel of $50 \times 75 \mathrm{~cm}^{2}$ cross-section and approximately $2 \mathrm{~m}$ length. For each value of the model speed, the experiment is repeated between 20 and 60 times, to obtain an ensemble estimate of the drag. The instantaneous model velocity $U_{M}$ is monitored with a high-speed camera (cam 4 in Fig. 1).

Helium-filled soap bubbles, used as flow tracers (Bosbach et al. 2009), are illuminated by a high-speed laser over a domain of $40 \times 40 \times 3 \mathrm{~cm}^{3}$. The interrogation volume of $96^{3}$ voxels corresponds to $4.4^{3} \mathrm{~cm}^{3}$ and velocity
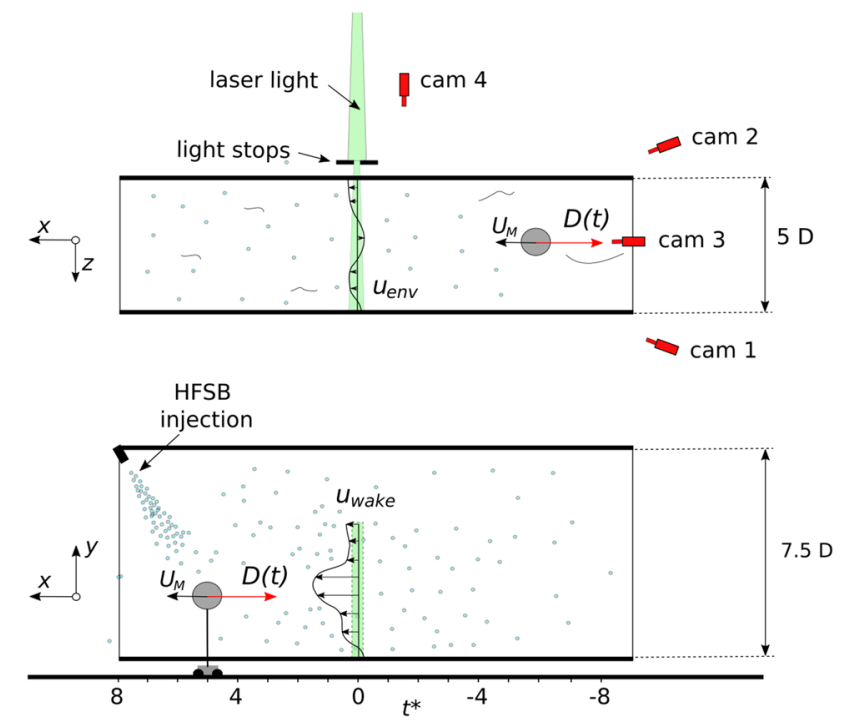

Fig. 1 Schematic top view of the experimental apparatus at $t^{*}=-6$ (top) and a side view at $t^{*}=5$ (bottom)

vectors are returned with a spacing of $11 \mathrm{~mm}$. Images are acquired at $500 \mathrm{~Hz}$ and the tomographic objects are reconstructed using every 5 th image with the sequential motion tracking enhancement technique (SMTE, Lynch and Scarano 2015) to enhance the PIV accuracy. A nondimensional time is defined as $t^{*}=t U_{M} / d$ where $t^{*}=0$ indicates the moment when the centre of the sphere is passing through the measurement station $(x=0)$. The flow conditions before the passage of the sphere are evaluated around $t^{*}=-6$. After the transit, the wake velocity is sampled over the time range $5<t^{*}<7$, as this allows the drag to be reliably evaluated without accounting for the pressure term in the momentum balance (Terra et al. 2017). A schematic view of the setup is depicted in Fig. 1, with the sphere prior to $\left(t^{*}=-6\right.$, top) and after the transit $\left(t^{*}=5\right.$, top).

Some air motion is introduced inside the channel, among others, by the HFSB seeding procedure. Therefore, the sphere encounters a fluid environment that is never perfectly stagnant. This aspect needs to be considered when calculating the momentum imparted by the sphere to the air during its transit and it is illustrated in Fig. 1 where the streamwise velocity component of the fluid is depicted, before (top) and after (bottom) the passage of the model, $u_{e n v}$ and $u_{\text {wake }}$, respectively.

The instantaneous drag force $D$, resulting from the relative motion between a model and a surrounding incompressible fluid, can be obtained by invoking the conservation of momentum in a control volume containing the object. Assuming the control volume extends sufficiently far into the freestream, the drag force can be expressed in terms 
of downstream surface integrals only (e.g., Rival and Van Oudheusden 2017):

$D(t)=-\rho \partial \partial t \iint_{S_{\text {wake }}} x u d S+\rho \iiint_{S_{\text {wake }}}\left(U_{\infty}-u\right) u d S+\iiint_{S_{\text {wate }}}\left(p_{\infty}-p\right) \mathrm{d} S$

where $\rho$ is the density, $u$ is the streamwise velocity component and $p$ the static pressure of the fluid. The freestream velocity and pressure are $U_{\infty}$ and $p_{\infty}$, respectively, and $S_{\text {wake }}$ is the downstream surface (orthogonal to $U_{\infty}$ ) of the control volume with $x$ the streamwise distance measured from any fixed frame of reference. The conservation of mass reduces the first integral at the right-hand-side of the equation to zero for stationary inflow conditions. In addition, the contribution of the pressure term is neglected, because it provides a contribution to the overall aerodynamic drag below $2 \%$ after $t^{*}>5$ (Terra et al. 2017). Hence, the expression for the drag force is largely simplified:

$D(t)=\rho \iint_{S_{\text {meas }}}\left(U_{\infty}-u\right) u \mathrm{~d} S$

This equation holds in the frame of reference moving with the model. The measurement instead are conducted in a fixed frame of reference (that of the laboratory) and, hence, the drag is rewritten in terms of $u_{\text {env }}$ and $u_{\text {wake }}$ (see Fig. 1):

$D(t)=\rho \iint_{S_{\text {meas }}}\left(u_{\text {env }}-u_{\text {wake }}\right)\left(u_{\text {wake }}-U_{M}\right) \mathrm{d} S$

where $S_{\text {meas }}$ is the fixed measurement cross-sectional area and $U_{\mathrm{M}}$ is the measured model velocity, which is assumed to be stationary. For each single transit, the drag of the model $\bar{D}_{\text {single }}$ is computed as the time-average drag over the range $5<t^{*}<7$. Consecutively, a multi-passage average drag $\bar{D}_{\text {multi }}$ is computed from the ensemble $\bar{D}_{\text {single }}$ obtained from repeated measurements at the same model velocity. The drag due to the strut estimated according to Terra et al.
(2017) amounts to approximately $14 \%$ of the total drag and is subtracted from it. A correction factor of 0.96 to the aerodynamic drag is applied to account for the model blockage of $2 \%$ by assuming continuous model blockage (Moradian et al. 2009).

Application of the wake rake technique in wind tunnels typically does not require a detailed measurement of the inflow conditions as the freestream velocity can be assumed uniform. In contrast, for towed experiments, the model may travel into a not fully stagnant environment. The impact of the non-stagnant nature of the flow prior to the passage on the resulting drag force is evaluated (Fig. 2). When the environment velocity is neglected (Fig. 2-left, blue symbols), the expected quadratic increase in drag is not observed, resulting in an RMS error $\varepsilon_{R M S}=1.5 \mathrm{mN}$ w.r.t. a quadratic fit of the five data points. Instead, accounting for the environment velocity yields values of the aerodynamic drag (Fig. 2-left, black symbols) in good agreement with the theoretical quadratic scaling with the model velocity (red-dashed line represents quadratic fit through the data points: $D=0.00177 U_{\mathrm{M}}{ }^{2}$ ) with an $\varepsilon_{R M S}=0.16 \mathrm{mN}$. The uncertainty of the multi-passage average drag is computed at $95 \%$ confidence level, conservatively using one uncorrelated drag value per integer value of $t^{*}$. The uncertainty, depicted by the size of the error bars, is reduced from above $20 \%$ to below $7 \%$ of the mean drag value when accounting for the environmental velocity, hence increasing the precision of the wake rake approach. The uncertainty of the aerodynamic drag includes the drag variations stemming from the changing density and velocity of the model from run to run. The uncertainty of drag coefficient, instead, does not, which is depicted in Fig. 2-right, and its relative uncertainty, therefore, is approximately $4 \%$ below that of the drag.

The flow conditions prior to the model passage changed from day to day during the experiment, which explains the variation between the corrected and uncorrected aerodynamic drag in Fig. 2-left over the different model speeds. Figure 3-left depicts this environmental velocity in terms of the spatial distribution of time-average velocity and its
Fig. 2 Multi-passage average drag $\bar{D}_{\text {multi }}$ (left) and drag coefficient $C_{D \text { multi }}$ (right) at five different model velocities assuming $u_{\text {env }}=0$ (blue symbols) and using the measured environment velocity $u_{e n v}$ upstream of the model transit (black symbols). The red-dashed lines represent a fit through the latter set of data points: $D=0.00177 U_{M}^{2}$ (left) and $C_{D}=0.365$ (right)
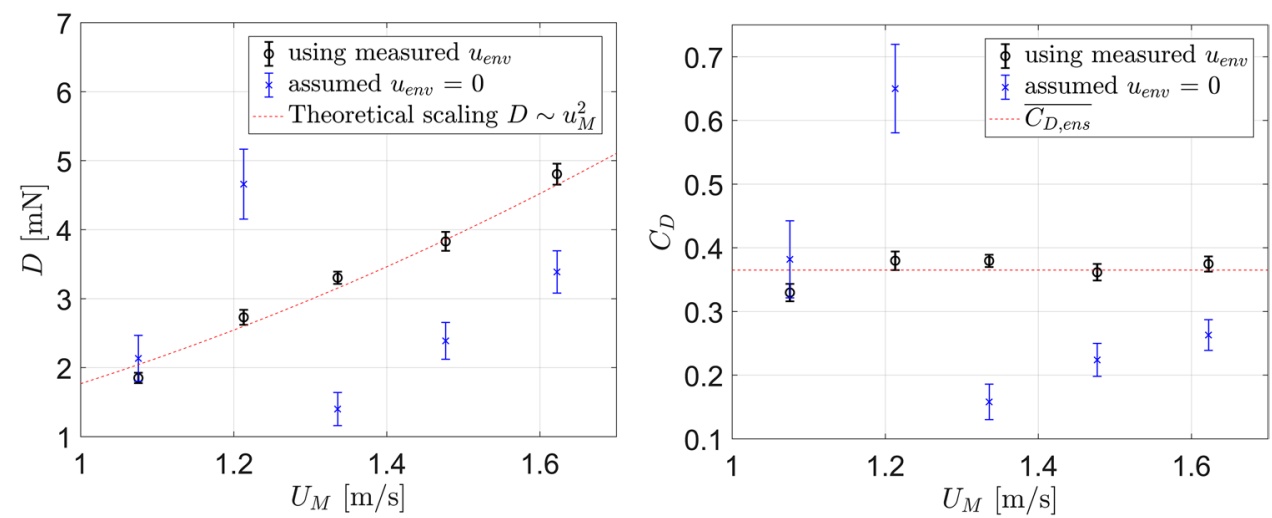

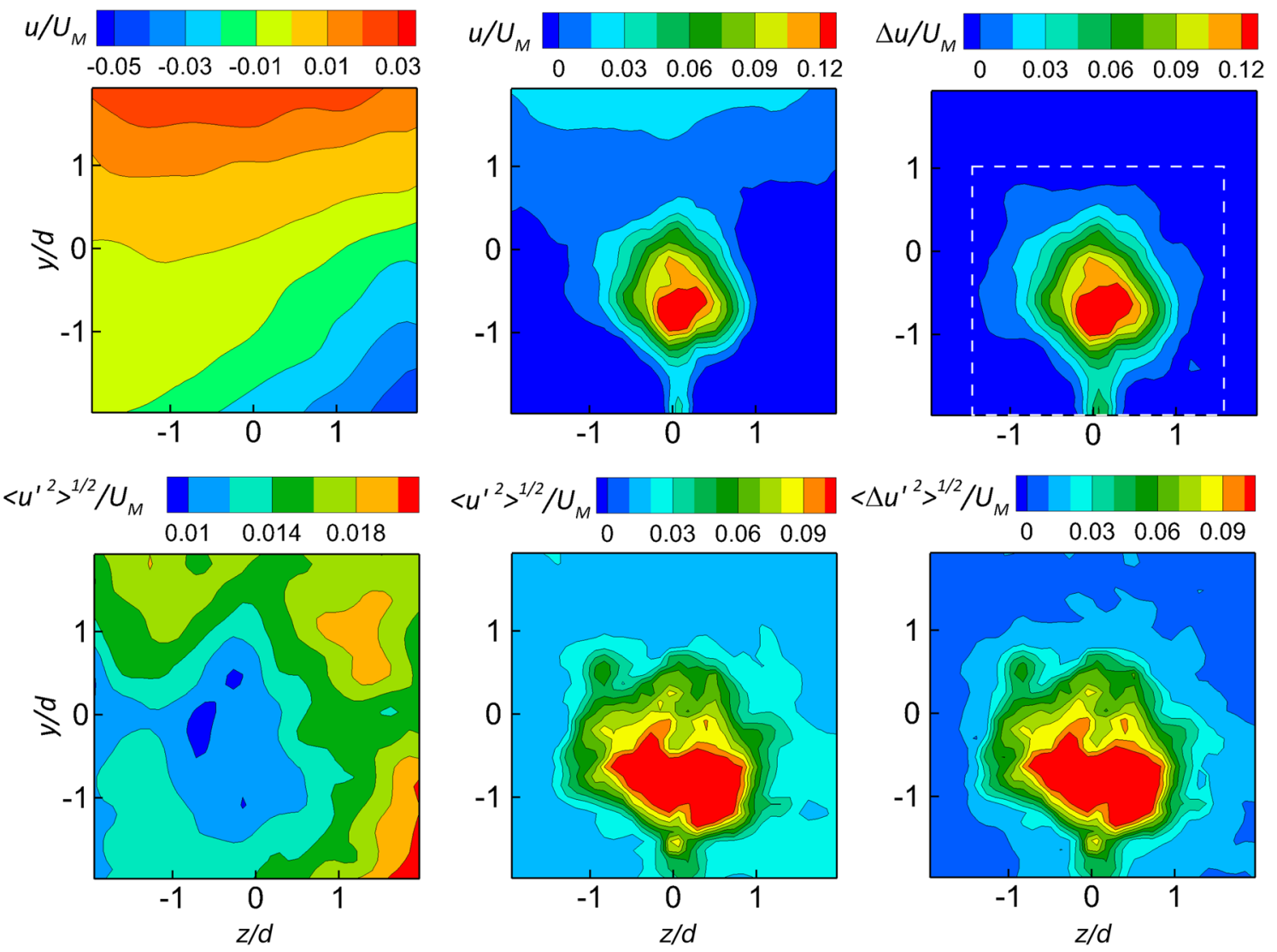

Fig. 3 Spatial distribution of the ensemble average streamwise velocity (top) and its fluctuations (bottom). Velocity prior to the passage of the model at $t^{*}=-6, u_{\text {env }}$ (left), and after five diameters $\left(t^{*}=5\right)$,

fluctuations, obtained from 53 repeated experiments at a model speed of $1.34 \mathrm{~m} / \mathrm{s}$. The results at the other speeds are omitted for reasons of conciseness. The time-averaged velocity distribution (Fig. 3, top-left) depicts a streamwise velocity up to $4 \%$ of the model velocity, which corresponds to up to $20 \%$ of the streamwise velocity measured in the wake of the model (Fig. 2, top-middle). Hence, for a correct estimate of the drag value, the streamwise velocity deficit $\Delta u=u_{\text {wake }}-u_{e n v}$ must be considered, which is illustrated in Fig. 3, top-right. In the latter, the velocity difference is brought close to zero almost everywhere outside of the sphere wake, except for the strut wake region. Fluctuations in the environment prior to the passage appear relatively uniform and limited to approximately $1.5 \%$ of the model velocity (Fig. 3, bottom-left). The fluctuations in the wake (Fig. 3, bottom-middle) are concentrated around the cross-section of the model. Subtracting the initial velocity of the environment, the level of the fluctuations outside of the wake is reduced considerably (Fig. 3, bottom-right), which further clarifies the increased precision of the PIV wake rake when $u_{e n v}$ is accounted for. $u_{\text {wake }}$ (middle). Net velocity deficit, $\Delta u=u_{\text {wake }}-u_{\text {env }}$ (right) for a model speed of $1.34 \mathrm{~m} / \mathrm{s}$

To identify the additional sources of uncertainty of the PIV wake rake technique, the time series of the drag coefficient in the interval $5<t^{*}<7$ for ten different passages of the model are depicted in Fig. 4, left. A second-order polynomial time filter, using a kernel of five samples $\left(\Delta t^{*}=0.67\right)$,

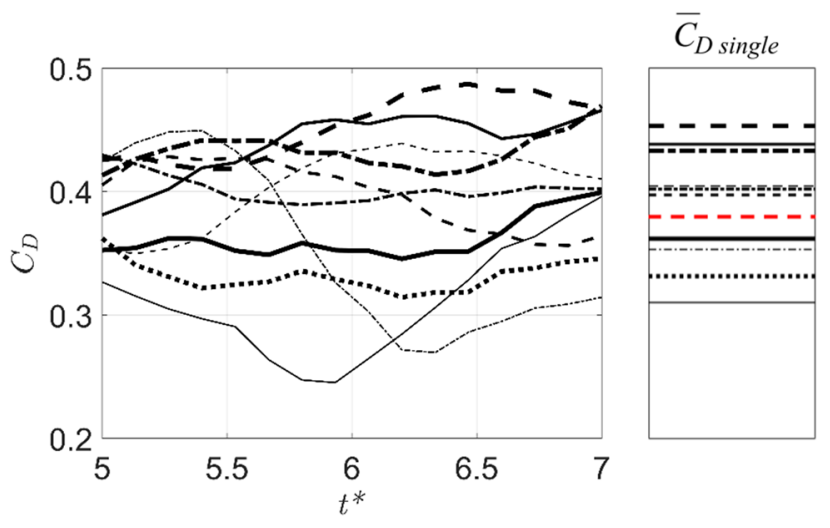

Fig. 4 Temporal evolution of the measured drag coefficient from ten individual model passages (left) and its value averaged in the interval $5<t^{*}<7$. The ensemble average value is given in red (right) 
has been applied to the underlying time-resolved velocity fields to reduce small-scale fluctuations of $C_{D}$ for better readability of the large-scale variations. The dispersion of the data encompasses minimum and maximum values of $C_{D}$ from 0.25 to 0.5 , respectively.

For a single passage, variations of the drag coefficient up to 0.15 are observed. The latter are ascribed to the unsteady behaviour of the wake, thus leading to a time-varying drag coefficient. Considering that the fluctuations of largest amplitude are observed over a time scale of approximately $T^{*}=2$ (corresponding to a Strouhal number of 0.5 ) an observation time of the order of several periods would be required to achieve statistical convergence of the drag coefficient from a single passage. For each individual passage, the standard deviation of the (instantaneous) drag coefficient, $C_{D}$, is computed with respect to the time-average of the specific measurement, $\bar{C}_{D \text { single }}$ (Fig. 4 , right). The ensemble average of these standard deviations is equal to $\sigma_{C D \text {,single }}=0.026$, exceeding the unsteady fluctuation of the $C_{D}$ of a sphere reported in the literature: Norman and McKeon (2011) measure $\sigma_{C D} \sim 0.02$ at $R e=50,000$ and the numerical work of Constantinescu and Squires (2003) reports $\sigma_{C D} \sim 0.017$ at $R e=10,000$. This over-prediction is largely attributed to the assumption of stationary flow prior to the model passage.

Second, considerable variations of $C_{D}$ are observed among different model passages, as can be seen from the comparison of the time-average drag coefficients from different runs (Fig. 4, right). Such variations are mainly ascribed to the variability of the experimental conditions (mainly the environment velocity and its unsteady behaviour) among different runs. In particular, Eq. 1 is valid under the assumption that the environment velocity field is stationary during the observation time $\left(-6<t^{*}<7\right)$. The uncertainty associated with the variability of experimental conditions is quantified from the standard deviation of the average drag coefficients $\overline{C_{D \text { single }}}$, and is equal to $2 \sigma_{C D \text {,multi }}=0.108$. This uncertainty is considered as an upper-bound of the drag coefficient resolution of the single-passage PIV wake rake for transiting models. Decreasing this uncertainty value is likely possible taking into account longer measuring times (increasing the range of $t^{*}$ ). Considering that the average drag coefficient $\overline{C_{D \text { single }}}$ is computed from $N=53$ passages of the sphere, the uncertainty of the former at $95 \%$ confidence level is $2 \sigma_{C D \text {,multi }} / \sqrt{N}=0.015$.

Both the drag coefficient resolution of the single-passage measurement and the uncertainty of the multi-passage approach may be decreased first through better flow conditioning, thus reducing the temporal fluctuations of $u_{e n v}$, and second by decreasing the size of the wake plane by excluding the area that does not result from the interaction between the model and fluid. For example, when the integration area is reduced to the rectangular region indicated by the dashed line in Fig. 3, top-right $(-2<y / D<1.5 ;-1.5<z / D<1.5)$, the uncertainty of the multi-passage average drag coefficient decreases by $10 \%$.

A third source of uncertainty may be introduced by possible systematic errors in the experiments, which can be evaluated from the drag coefficient obtained over repeated measurements at different model speed presented in Fig. 2, right. As mentioned before, in this narrow range of Reynolds number, the drag coefficient of the sphere is expected to be constant. Values of average drag coefficient between $0.33<{\overline{C_{D}}}_{\text {multi }}<0.38$ are obtained, with an ensemble average drag coefficient of $\overline{C_{\text {Dens }}}=0.365$ (red-dashed line in Fig. 2, right). The drag resolution of the PIV wake rake is estimated as the standard deviation of the average drag coefficients at different model speeds with respect to the ensemble average value:

$\Delta C_{D}=\sqrt{\frac{1}{N_{M}-1} \sum_{i=1}^{N_{M}}\left(\overline{C_{D, \text { multi }, i}}-\overline{C_{D, \text { ens }}}\right)^{2}}$

where $N_{M}$ is the amount of different model speeds that are considered (here $N_{M}=5$ ). When the drag resolution is computed considering the five model velocities, the value $\Delta C_{D}=0.021$ or 21 drag counts is obtained (see also Table 1 ). When the drag coefficients are computed under the assumption of stagnant flow prior to the passage of the model $\left(u_{e n v}=0\right)$, the accuracy of the measurement is strongly reduced (see Fig. 2, right) and the drag resolution is equal to $\Delta C_{D}=0.193$ (193 drag counts). This again clearly illustrates the necessity of measuring the flow prior to the passage to estimate the drag force accurately.

Based on the results of this work, the following is concluded: (1) having flow conditions that are not fully stagnant, measurement of the spatial distribution of the flow velocity prior to the passage of the model is necessary for accurate drag estimations via the PIV wake rake approach of transiting bluff bodies; (2) the tomo-PIV wake rake approach

Table 1 Comparison between the drag resolution of the PIV wake rake of a transiting model to that of other techniques

\begin{tabular}{ll}
\hline & $\begin{array}{l}\text { Drag resolution } \\
\text { (drag counts) }\end{array}$ \\
\hline Stationary model in wind tunnel & \\
Force balance (Philipsen et al. 2004) & 0.5 \\
Pitot-tube wake rake (Brune 1994) & 1 \\
Transiting model & \\
PIV wake rake (current study) & 21 \\
Coast-down method (Petrushov 1998) & 12 \\
Torque measurement (Bouillod et al. 2015) & 10 \\
\hline
\end{tabular}


returns the average drag coefficient from multiple passages of transiting objects with a drag resolution in the order of 20 drag counts.

It should be noticed that, with a drag resolution of about 20 drag counts, the PIV wake rake for transiting models remains a relatively coarse instrument compared to established techniques used in the laboratory environment (see Table 1 for a comparison), but it becomes comparable to in-field approaches, such as the coast-down and torque measurement method. The additional contribution of the PIV wake rake is the introduction of fluid-dynamic information on the wake structure, of potential interest in the understanding of the flow physics and drag generation. It is expected that the current approach may be further perfected especially with control of environment conditions prior to the passage and possibly with more advanced modelling of the wake region.

Acknowledgements This work is partly funded by the TU Delft Sports Engineering Institute and the European Research Council Proof of Concept Grant "Flow Visualization Based Pressure" (No. 665477). Alexander Spoelstra is acknowledged for the support during experiments.

Open Access This article is distributed under the terms of the Creative Commons Attribution 4.0 International License (http://creativeco mmons.org/licenses/by/4.0/), which permits unrestricted use, distribution, and reproduction in any medium, provided you give appropriate credit to the original author(s) and the source, provide a link to the Creative Commons license, and indicate if changes were made.

\section{References}

Bosbach J, Kühn M, Wagner C (2009) Large scale particle image velocimetry with helium filled soap bubbles. Exp Fluids 46:3 539-547. https://doi.org/10.1007/s00348-008-0579-0

Bouillod A, Pinot J, Froncioni A, Grappe F (2015) Validity of track aero system to assess aerodynamic drag in professional cyclists. J Sci Cycling 4(2):7-8

Brownlie L, Aihara Y, Carbo J, Harber E Jr, Henry R, Ilcheva I, Ostafichuk P (2016) The use of vortex generators to reduce the aerodynamic drag of athletic apparel. Proc Eng 147:20-25

Brune GW (1994) Quantitative low-speed wake surveys. J Aircraft 31(2):249-255

Constantinescu GS, Squires KD (2003) LES and DES investigations of turbulent flow over a sphere at $R e=10,000$. Flow Turbul Combust $70:: 267-298$
De Kat R, Bleischwitz R (2016) Towards instantaneous lift and drag from stereo-PIV wake measurements. In: Proceedings of the 18th international symposium on application of laser and imaging techniques to fluid mechanics, Lisbon

Jones BM (1936) Measurement of profile drag by the pitot-traverse method. ARC R\&M 1688

Lynch KP, Scarano F (2015) An efficient and accurate approach to MTE-MART for time-resolved tomographic PIV. Exp Fluids 56:66. https://doi.org/10.1007/s00348-015-1934-6

Maskell EC (1973) Progress towards a method for the measurement of the components of the drag of a wing of finite span. RAE technical report 72232

Moradian N, Ting DSK, Cheng S (2009) The effects of freestream turbulence on the drag coefficient of a sphere. Exp Therm Fluid Sci 33:460-471. https://doi.org/10.1016/j.expthermflusci.2008.11.001

Norman AK, McKeon BJ (2011) Unsteady force measurements in sphere flow from subcritical to supercritical Reynolds numbers. Exp Fluids 51:1439-1453. https://doi.org/10.1007/s0034 8-011-1161-8

Petrushov VA (1998) Improvement in vehicle aerodynamic drag and rolling resistance determination from coast-down tests. Proc Inst Mech Eng 212:369-380

Philipsen I, Hoeijmakers H, Alons HJ (2004) A new balance and airreturn line bridges for DNW-LLF models. NLR-TP-2004-315

Rival DE, Van Oudheusden B (2017) Load-estimation techniques for unsteady incompressible flows. Exp Fluids 58:20. https://doi. org/10.1007/s00348-017-2304-3

Scarano F, van Wijk C, Veldhuis LLM (2002) Traversing field of view and AR-PIV for mid-field wake vortex investigation in a towing tank. Exp Fluids 33:950-961. https://doi.org/10.1007/s0034 8-002-0516-6

Schlichting H (1979) Boundary-layer theory. McGraw-Hill, New York

Selig MS, Deters RW, Williamson GA (2011) Wind tunnel testing airfoils at low Reynolds numbers. In: AIAA 2011-875, 4-7 January, Orlando, FL

Terra W, Sciacchitano A, Scarano F (2017) Aerodynamic drag of a transiting sphere by large-scale tomographic-PIV. Exp Fluids 58:83. https://doi.org/10.1007/s00348-017-2331-0

Van Oudheusden BW, Scarano F, Roosenboom EWM, Casimiri EWF, Souverein LJ (2007) Evaluation of integral forces and pressure fields from planar velocimetry data for incompressible and compressible flows. Exp Fluids 43:153-162. https://doi.org/10.1007/ s00348-007-0261-y

Von Carmer CF, Heider A, Schröder A, Konrath R, Agocs J, Gilliot A, Monnier JC (2008) Evaluation of large-scale wing vortex wakes from multi-camera PIV measurements in free-flight laboratory. Part Image Velocim Top Appl Phys 112:377-394

Publisher's Note Springer Nature remains neutral with regard to jurisdictional claims in published maps and institutional affiliations. 